\title{
Quadriceps femoris muscle fatigue in patients with knee osteoarthritis
}

\author{
This article was published in the following Dove Press journal: \\ Clinical Interventions in Aging \\ 12 August 2013 \\ Number of times this article has been viewed
}

\section{Elboim-Gabyzon' \\ N Rozen ${ }^{2}$ \\ Y Laufer'}

'Physical Therapy Department, Faculty of Social Welfare and Health Sciences, University of Haifa, Haifa, Israel;

${ }^{2}$ Emek Medical Center, Afula, Israel
Correspondence:

M Elboim-Gabyzon

Department of Physical Therapy,

Faculty of Social Welfare and Health

Sciences, Haifa University, Mount Carmel,

Haifa 31905, Israel

Tel +972 545968943

E-mail michal.elboim@gmail.com

\begin{abstract}
The purpose of this study was to characterize quadriceps femoris muscle fatigue of both lower extremities in patients with knee osteoarthritis (OA). Sixty-two subjects (mean age 68.2 years, standard deviation $[\mathrm{SD}] \pm 7.9$ years) with knee OA participated in the study. Significantly higher knee pain was reported in the involved knee than in the contralateral knee, as determined by a visual analog scale. Significant differences were demonstrated between the lower extremities in terms of maximal voluntary isometric contraction, in favor of the less involved leg $(P=0.0001)$. In contrast, the degree of fatigue of the quadriceps femoris muscle, as measured by the decrement in force production following ten repeated contractions, was significantly higher in the contralateral leg $(P=0.0002)$. Furthermore, normalization of the fatigue results to the first contraction yielded a similar result $(P<0.0001)$. Similar results were noted when analysis was performed separately for subjects whose involvement was unilateral or bilateral. The results indicate that, irrespective of the initial strength of contraction, the rate of muscle fatigue in the contralateral leg is significantly higher than in the involved leg. Hypotheses for these unexpected results are suggested. Rehabilitation of patients with knee OA should focus on increasing quadriceps muscle strength and endurance for both lower extremities.
\end{abstract}

Keywords: knee, osteoarthritis, fatigue, quadriceps femoris muscle

\section{Introduction}

Decline in quadriceps femoris muscle strength is common in individuals with knee osteoarthritis (OA), ${ }^{1}$ and is due primarily to disuse atrophy and impaired ability of the central nervous system to recruit existing motor units despite maximum conscious effort. ${ }^{2}$ In contrast to strength, muscle fatigue, which is defined as a temporary loss or decrease in force-generating ability due to recent contractions, ${ }^{3}$ has rarely been examined in patients with knee OA. Although methods to assess muscle fatigue in patients with knee OA have been validated, ${ }^{4,5}$ to the best of our knowledge, the only direct measure of muscle fatigue in this population was reported by Fisher et al. ${ }^{6}$ Their study of 15 male subjects $(67.6 \pm 6.1$ years) with knee OA demonstrated that muscle strength and endurance were 50\% lower than in an age-matched control group.

Muscle fatigue has important clinical implications due to the significant role of quadriceps muscle endurance for functional capabilities in the activities of daily living, such as walking and climbing stairs. As these capabilities are particularly affected in patients with knee OA, ${ }^{7}$ there is a need to expand our understanding of the characteristics of quadriceps muscle fatigue in this population.

The scope of the literature regarding quadriceps muscle performance in patients with knee pathologies has changed in recent years, shifting from a previous focus only 
on the symptomatic knee to a broader view of both lower extremities. ${ }^{8}$ This is probably due to the fact that patients often demonstrate symptoms in both legs. Furthermore, even when one leg is asymptomatic, cartilage degeneration might already exist and may be confirmed by radiography. ${ }^{9,10}$ In addition, muscle dysfunction in one leg may be a factor in hastening the progression of joint breakdown in the contralateral leg. ${ }^{11,12}$ Accordingly, there is justification for evaluating quadriceps muscle performance and, particularly, quadriceps muscle fatigue of both lower extremities in patients with knee OA.

The purpose of this study was to compare the fatigability of the quadriceps muscle by assessing isometric contraction properties of both lower extremities in patients with knee OA. It was expected that the affected (or more affected) leg would have weaker maximal voluntary isometric contraction (MVIC) and a higher rate of fatigue compared to the contralateral leg.

\section{Study methodology Subjects}

All patients referred to an orthopedic outpatient physical therapy clinic between September 2008 and December 2009 with the diagnosis of idiopathic knee OA were screened by a physical therapist from the clinic using an eligibility checklist. This descriptive study is a subset of a larger cohort randomized controlled trial. ${ }^{13}$

Inclusion criteria were: (1) radiographic evidence of knee OA at a grade $\geq$ II, according to the Kellgren and Lawrence classification; ${ }^{14}$ (2) age above 50; (3) compliance matching with the classification of the American College of Rheumatology; ${ }^{15}$ (4) knee pain for at least 3 months, with pain presenting at least 3 days a week during the last month; (5) ability to ambulate independently for at least 10 meters; and (6) ability to follow simple instructions. Exclusion criteria were: (1) use of a pacemaker; (2) history of cardiovascular, neurological, or orthopedic problems that could affect functional performance; (3) previous knee surgery other than arthroscopy; (4) injections to the knee joint during the previous 6 months; and (5) change in pain medication in the previous month.

The study coordinator explained the purpose and procedures of the study to the eligible participants. The study was approved by the Helsinki committee of the Emek Medical Center, and all subjects signed an informed consent form prior to assessment.

\section{Procedures}

The subjects completed questionnaires regarding their knee pain intensity, followed by an assessment of their quadriceps muscle performance.

\section{Pain assessment}

Subjects evaluated their level of knee pain during the previous week on a visual analog scale (VAS), with 0 representing no pain and 10 representing the most severe pain (intraclass correlation coefficient $[\mathrm{ICC}]=80){ }^{16}$

\section{Assessment of the quadriceps femoris muscle isometric contraction}

A computerized myometry system (Quantitative Muscle Assessment [QMA] system; Aeverl Medical, Gainesville, GA, USA) was used for all quadriceps muscle assessments. Test-retest reliability for this apparatus was previously determined in our laboratory $(\mathrm{ICC}=0.89) .{ }^{17}$ Assessment included the following tests: (1) quadriceps muscle MVIC test; (2) quadriceps muscle fatigue test. The two tests were implemented in the same order for all patients, and there was a 5-minute rest period between tests.

Both knees were tested, with the uninvolved or less painful leg (in the case of bilateral knee involvement) tested first, followed by the involved leg. All quadriceps muscle assessments were carried out while the subjects were seated on a straight-back chair, with hips flexed at $90^{\circ}$ and knees flexed as close to $90^{\circ}$ as could be tolerated, a protocol previously used for muscle assessment with knee OA. ${ }^{18-20}$ The thigh, chest, and pelvis were stabilized using inelastic straps with hook-and-loop closures to prevent substitution movements by the body, and the arms were positioned across the chest. The ankles were attached to a padded strap placed $2 \mathrm{~cm}$ above the lateral malleolus and connected to a high-tension wire with a strain gauge (SM250 force transducer). The system's software provided summary data of force and force-integral outputs at a $50 \mathrm{~Hz}$ data acquisition rate.

\section{MVIC}

The subjects first performed a 5-minute warm-up on a stationary bicycle with no resistance. The subjects were then seated in the testing apparatus and, following a 5-minute rest period, performed three to five isometric contractions against the strain gauge at $50 \%, 75 \%$, and $100 \%$ of their perceived MVIC. ${ }^{21}$ To achieve this goal, the subjects were instructed to "push the strain gauge at $50 \%, 75 \%$, and $100 \%$ of what you perceive your maximum force to be." The intensity of these contractions was monitored on the screen of the computer by the researcher to ensure that the subjects understood the instructions. The data for these preliminary contractions were excluded from the analysis.

Following a second 5-minute rest break, participants performed three consecutive brief ( $\sim 5$-second) MVICs of the quadriceps, each separated by 90 seconds of rest. 
The investigators offered standardized verbal encouragement throughout the testing protocol. An additional contraction was attained when one of the consecutive MVICs differed by more than $5 \%$ from the others. The mean of the three MVIC trials was utilized for subsequent calculations. In addition, the values were normalized to the subjects' weight.

\section{Fatigue test procedure}

The subjects were asked to perform ten consecutive MVICs of the quadriceps muscle, each lasting 10 seconds. ${ }^{22,23}$ Two indexes were utilized in order to quantify quadriceps muscle fatigue in each leg: (1) the fatigue index was calculated by subtracting the value of the tenth MVIC from the value of the first MVIC; (2) the fatigue index normalized to the first contraction. For the fatigue index, the first contraction was defined as $100 \%$, and all other contractions were defined as a percentage of this contraction. The index value was calculated as the tenth contraction (in percentage) subtracted from 100. Thus, in both indexes, lower numbers indicate greater fatigue.

\section{Statistical analyses}

The following variables were considered for analysis: VAS; MVIC; fatigue index; normalized fatigue index. Additionally, the muscle force and fatigue measures were normalized to body-weight. Descriptive statistics (means, standard deviations [SDs]) were calculated for all outcome measures. Normalized distribution was determined for all variables using the Shapiro-Wilk test. One-way analyses of variance (ANOVAs) were performed to determine differences between the involved leg and the contralateral leg with all subjects grouped together, as well as separately for subjects whose involvement was unilateral or bilateral. Since a large variability in body mass index (BMI) was observed, the analysis was repeated with the BMI as a covariant, for the entire group as well as for each group separately, for all outcome measures. Level of significance was set at $\alpha=0.05$. Statistical analyses were performed using JMP (SAS Institute, Cary, NC, USA).

\section{Results}

Of the 134 patients with a diagnosis of knee OA who were considered as potential candidates for the study, 62 subjects (51 females, eleven males) were found to be eligible to take part in the study based on the inclusion/exclusion criteria. The characteristics of the 62 subjects are presented in Table 1.

Forty-three subjects had a bilateral leg involvement and 19 subjects had a unilateral involvement. In case of bilateral involvement, the more painful leg was considered
Table I The subjects' characteristics $(n=62)$

\begin{tabular}{ll}
\hline Characteristic & Value \\
\hline Age (years): mean \pm SD (range) & $68.2 \pm 7.9(54-83)$ \\
Weight (kg): mean \pm SD (range) & $79.4 \pm 15.6(48-120)$ \\
Height (cm): mean \pm SD (range) & $160.9 \pm 7.4(148-176)$ \\
BMI (weight/height $\left.{ }^{2}\right):$ mean \pm SD (range) & $30.9 \pm 5.8$ (I9.5-47.3) \\
Sex (women:men) & $51: 11$ \\
Duration of knee osteoarthritis (years): & $4.3 \pm 5.5(0.17-25)$ \\
mean \pm SD (range) & \\
Use of walking device (yes:no) & $6: 56$ \\
Participation in regular physical activity (yes:no) & $14: 48$ \\
Involvement of both legs (yes:no) & $43: 19$ \\
\hline
\end{tabular}

Abbreviations: SD, standard deviation; BMI, body mass index.

the involved leg. The muscle performance results of the entire group, as well as the separate results of the group with unilateral involvement and the group with bilateral involvement, are presented in Table 2 . As the results of the statistical analysis of all the muscle performance variables normalized for weight did not differ from the results of the non normalized values, only the non normalized values are presented (see Table 2). Additionally, analysis with BMI as a covariant determined no significant effect of BMI on any of the outcome measures.

\section{Pain intensity}

The mean (SD) pain intensities of the entire group of the involved knee and contralateral leg were $7.2 \mathrm{~cm}$ (1.95) and $3.2 \mathrm{~cm}$ (2.86), respectively. The overall difference between the legs with respect to pain intensity was statistically significant $(P<0.0001)$. In the group with unilateral involvement, mean (SD) VAS of the involved leg was $6.3 \mathrm{~cm}$ (2.14), with no pain reported in the contralateral leg. In the group with bilateral involvement, mean (SD) VAS of the more affected sides and the less affected side were $7.6 \mathrm{~cm}$ (1.66) and $4.6 \mathrm{~cm}$ (2.26), respectively. In both subgroups with the unilateral and bilateral leg involvement the difference between the legs with respect to pain intensity was statistically significant $(P<0.0001)$.

\section{Quadriceps femoris muscle isometric contraction performance}

The $90^{\circ}$ knee flexion position was well tolerated by all subjects. The MVIC in the involved leg of the entire group was significantly lower than in the contralateral leg $(P=0.0001)$. Similar statistical differences were observed in the groups with unilateral and with bilateral leg involvement (see Table 2).

\section{Fatigue test performance}

The mean $( \pm \mathrm{SD})$ force of the first and tenth contractions in the fatigue tests of the entire group, and of the groups 
Table 2 Quadriceps femoris muscle isometric contraction performance of both legs (mean \pm SD) and comparison between the legs $(P$-value $)$ of the entire group and of the groups with unilateral and bilateral leg involvement

\begin{tabular}{|c|c|c|c|c|c|c|c|c|c|}
\hline & \multicolumn{3}{|c|}{ The entire group $(n=62)$} & \multicolumn{3}{|c|}{ Unilateral involvement $(n=19)$} & \multicolumn{3}{|c|}{ Bilateral involvement $(n=43)$} \\
\hline & $\begin{array}{l}\text { Involved } \\
\text { leg }\end{array}$ & $\begin{array}{l}\text { Uninvolved } \\
\text { leg }\end{array}$ & $P$-value & $\begin{array}{l}\text { Involved } \\
\text { leg }\end{array}$ & $\begin{array}{l}\text { Uninvolved } \\
\text { leg }\end{array}$ & $P$-value & $\begin{array}{l}\text { Involved } \\
\text { leg }\end{array}$ & $\begin{array}{l}\text { Uninvolved } \\
\text { leg }\end{array}$ & $P$-value \\
\hline MVIC (kg) & $14.4 \pm 6.3$ & $16.5 \pm 6.6$ & 0.0001 & $\mid 4.1 \pm 6.1$ & $16.4 \pm 6.2$ & 0.02 & $14.5 \pm 6.5$ & $16.6 \pm 6.9$ & 0.003 \\
\hline Fatigue index (kg) & $-1.9 \pm 2.8$ & $-4.3 \pm 3.0$ & 0.0002 & $-2.0 \pm 4.1$ & $-4.6 \pm 3.5$ & 0.04 & $-1.9 \pm 1.9$ & $-4.2 \pm 2.8$ & $<0.000$ I \\
\hline $\begin{array}{l}\text { Normalized fatigue } \\
\text { index (\%) }\end{array}$ & $-14.4 \pm 14.7$ & $-24.3 \pm 14.5$ & $<0.000 \mathrm{I}$ & $-13.7 \pm 15.7$ & $-21.3 \pm 11.9$ & 0.12 & $-14.8 \pm 14.5$ & $-25.7 \pm 15.4$ & 0.0006 \\
\hline
\end{tabular}

Abbreviations: SD, standard deviation; MVIC, maximal voluntary isometric contraction.

with unilateral and bilateral involvement, are presented in Table 3. The degree of fatigue of the quadriceps muscle, as indicated by the fatigue index of the entire group, was significantly greater in the contralateral leg than in the involved leg $(P=0.002)$ (see Table 2 and Figure 1$)$. This significant difference between the legs held true even when the fatigue index was normalized to the first contraction $(P<0.0001)$ (see Table 2 and Figure 2). This additional analysis was conducted as the first MVIC of the contralateral leg was significantly stronger than the first contraction of the involved leg (see above; $P=0.0001$ ). The separate analysis, by group, indicated significant leg difference in the two fatigue indexes in the groups with unilateral and with bilateral leg involvement $(P=0.04, P<0.0001$, respectively), except that in the group with unilateral involvement $(\mathrm{n}=19)$, the comparison between legs for the normalized fatigue index did not quite reach significance $(P=0.12)$.

\section{Discussion}

Significant differences were observed between the lower extremities in terms of MVIC of the quadriceps muscle in the entire group and in the subgroups with the unilateral and bilateral leg involvement, in favor of the less involved leg. In contrast, surprisingly, the degree of fatigue (decrement in force generating ability following recent repeated contractions) of the quadriceps muscle was significantly higher in the less involved or uninvolved knee. It appears these results have never been previously reported.

As the contralateral leg was significantly stronger to start with, in the overall group and in the subgroups, which could affect fatigue difference between legs, a normalization of the fatigue results was done to the first contraction. Yet, this analysis provided similar results which may indicate that irrespective of the initial strength of contraction, the rate of muscle fatigue in the contralateral leg is significantly higher than in the involved leg. The only exception to this rule was demonstrated in the normalized fatigue index for the subgroup of patients with unilateral involvement where the comparison between legs did not reach significance. However, this may be due to the small size of the group $(n=19)$, which did not provide sufficient power for the analysis. While it may be claimed that the greater fatigability of the less involved leg is due to a floor effect, the raw data (Table 3) indicate that this is not a likely cause for the observed results.

We can only hypothesize as to the causes of this unexpected phenomenon. It might be that the pain related to OA stimulates nociceptive afferents that decrease the initial maximal force produced, as indicated by a central activation failure during MVIC., ${ }^{213}$ Consequently, the lower reduction of muscle strength during the fatigue test observed in the involved limb could be the result of submaximal muscle contraction during the consecutive isometric contractions. This may prevent muscle overload during repeated contractions and therefore reduce maximal utilization of the metabolic and excitation-coupling abilities of the muscle, subsequently reflected in the observed lower degree of muscle fatigue.

Another possibility may be related to differences in the muscle fiber composition between the quadriceps muscle of the two extremities. Selective atrophy of type II fast-

Table 3 The mean $( \pm S D)$ force of the first and tenth contractions in the fatigue tests of the entire group and of the groups with unilateral and bilateral leg involvement

\begin{tabular}{|c|c|c|c|c|c|c|}
\hline & \multicolumn{2}{|c|}{ The entire group $(n=62)$} & \multicolumn{2}{|c|}{ Unilateral involvement $(n=19)$} & \multicolumn{2}{|c|}{ Bilateral involvement $(n=43)$} \\
\hline & Involved leg & Uninvolved leg & Involved leg & Uninvolved leg & Involved leg & Uninvolved leg \\
\hline First MVIC (kg) & $14.3 \pm 6.7$ & $17.2 \pm 6.6$ & $14.3 \pm 6.3$ & $17.7 \pm 6.7$ & $14.4 \pm 7.0$ & $16.9 \pm 6.7$ \\
\hline Tenth MVIC (kg) & $12.2 \pm 5.9$ & $12.8 \pm 5.12$ & $12.9 \pm 5.6$ & $13.4 \pm 4.7$ & $11.8 \pm 6.2$ & $12.5 \pm 5.4$ \\
\hline
\end{tabular}

Abbreviations: SD, standard deviation; MVIC, maximal voluntary isometric contraction. 


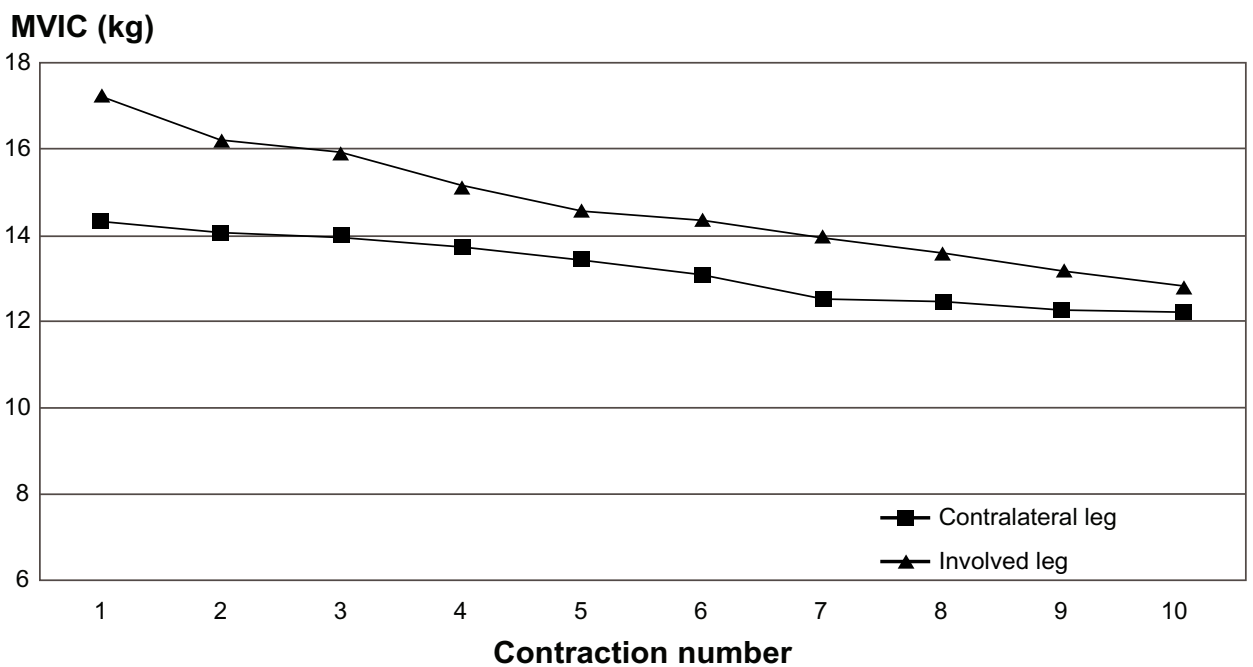

Figure I Mean strength of ten consecutive quadriceps femoris muscle maximal voluntary isometric contractions (MVICs) of both legs.

twitch fibers in quadriceps muscle has been repeatedly demonstrated in histological studies involving subjects with knee OA. ${ }^{24-26}$ This selective fiber atrophy is suggested to occur as a consequence of pain-related immobilization due to OA. ${ }^{27,28}$ Muscles with a higher percentage of fasttwitch fibers have been shown to fatigue more rapidly than muscles with a greater percentage of slow-twitch fibers. ${ }^{29}$ Thus, perhaps, the contralateral leg, which was significantly less painful than the involved leg, had a lower decline rate in type II fast-twitch fibers, resulting in a lower proportion of type I fatigue-resistant fibers. Accordingly, the asymmetric decline in type II fibers between the extremities rendered the quadriceps muscle of the involved leg more fatigue resistant. A similar rationale has been suggested for sex-related differences in quadriceps muscle fatigue in patients with knee OA. ${ }^{24} \mathrm{~A}$ higher proportion of slow, fatigue-resistant, type I fibers noted in women is suggested as the reason for the higher degree of fatigue observed in age-matched male subjects with knee $\mathrm{OA} ; ;^{30-32}$ however, further studies are needed to substantiate these speculative hypotheses.

\section{Limitations}

The current experimental design used dynamometry, which has been shown to be reliable and valid, and which is readily available in the clinical setting. ${ }^{33}$ However, in spite of standardized verbal encouragements, the results may have been affected by the subjects' motivation and pain, particularly during repeated contractions. ${ }^{34}$ Further studies with larger samples should examine whether the results are affected by the severity of OA and should include a healthy control group.

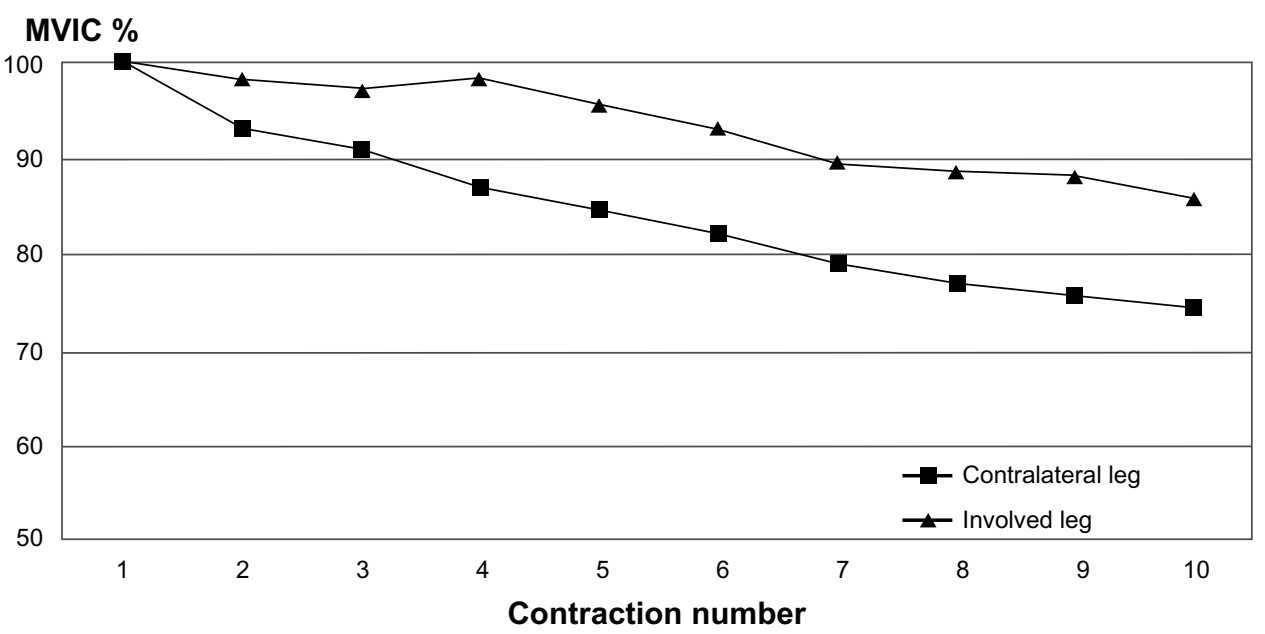

Figure 2 Mean strength of ten consecutive quadriceps femoris muscle maximal voluntary isometric contractions (MVICs) normalized to the first contraction of both legs. 


\section{Conclusion}

The results of this study indicate that, while the quadriceps muscle of the involved leg is weaker than the quadriceps muscle of the contralateral leg, the decrement in quadriceps muscle force production following repeated contractions - that is, muscle fatigue - is greater in the contralateral leg. It is recommended that the rehabilitation of patients with knee OA include exercises aimed at increasing quadriceps muscle endurance for both lower extremities.

\section{Disclosure}

The authors report no conflicts of interest in this work.

\section{References}

1. O'Reilly SC, Jones A, Muir KR, Doherty M. Quadriceps weakness in knee osteoarthritis: the effect on pain and disability. Ann Rheum Dis. 1998;57:588-94.

2. Lewek MD, Rudolph KS, Snyder-Mackler L. Quadriceps femoris muscle weakness and activation failure in patients with symptomatic knee osteoarthritis. J Orthop Res. 2004;22:110-115.

3. Aagaard P, Simonsen EB, Andersen JL, Magnusson P, Dyhre-Poulsen P. Increased rate of force development and neural drive of human skeletal muscle following resistance training. J Appl Physiol. 2002;93: 1318-1326.

4. Callaghan MJ, McCarthy CJ, Oldham JA. The reliability of surface electromyography to assess quadriceps fatigue during multi joint tasks in healthy and painful knees. J Electromyogr Kinesol. 2009;19: 172-180.

5. McCarthy CJ, Callaghan MJ, Oldham JA. The reliability of isometric strength and fatigue measures in patients with knee osteoarthritis. Man Ther. 2008;13:159-164.

6. Fisher NM, White SC, Yack HJ, Smolinski RJ, Pendergast DR. Muscle function and gait in patients with knee osteoarthritis before and after muscle rehabilitation. Disabil Rehabil. 1997;19:47-55.

7. Guccione AA, Felson DT, Anderson JJ, et al. The effects of specific medical conditions on the functional limitations of elders in the Framingham Study. Am J Public Health. 1994;84:351-358.

8. Pietrosimone BG, Hertel J, Ingersoll CD, Hart JM, Saliba SA. Voluntary quadriceps activation deficits in patients with tibiofemoral osteoarthritis: a meta-analysis. PM R. 2011;3:153-162; quiz 162.

9. Zeni JA Jr, Snyder-Mackler L. Early postoperative measures predict 1- and 2-year outcomes after unilateral total knee arthroplasty: importance of contralateral limb strength. Phys Ther. 2010;90:43-54.

10. Zhang Y, Jordan J. Epidemiology of osteoarthritis. Clin Geriatr Med. 2010;26:355-369.

11. Slemenda C, Brandt KD, Heilman DK, et al. Quadriceps weakness and osteoarthritis of the knee. Ann Intern Med. 1997;127:97-104.

12. Mikesky AE, Mazzuca SA, Brandt KD, Perkins SM, Damush T, Lane KA. Effects of strength training on the incidence and progression of knee osteoarthritis. Arthritis Rheum. 2006;55:690-699.

13. Elboim-Gabyzon M, Rozen N, Laufer Y. Does neuromuscular electrical stimulation enhance the effectiveness of an exercise programme in subjects with knee osteoarthritis? A randomized controlled trial. Clin Rehabil. 2013;27:246-257.

14. Kellgren JH, Lawrence JS. Radiological assessment of osteo-arthrosis. Ann Rheum Dis. 1957;16:494-502.

15. Altman RD. Criteria for classification of clinical osteoarthritis. J Rheumatol Suppl. 1991;27:10-12.
16. Boonstra AM, Schiphorst Preuper HR, Reneman MF, Posthumus JB, Stewart RE. Reliability and validity of the visual analogue scale for disability in patients with chronic musculoskeletal pain. Int $J$ Rehabil Res. 2008;31:165-169.

17. Laufer Y, Elboim M. Effect of burst frequency and duration of kilohertzfrequency alternating currents and of low-frequency pulsed currents on strength of contraction, muscle fatigue, and perceived discomfort. Phys Ther. 2008;88:1167-1176.

18. Berger MJ, McKenzie CA, Chess DG, Goela A, Doherty TJ. Quadriceps neuromuscular function and self-reported functional ability in knee osteoarthritis. J Appl Physiol. 2012;113:255-262.

19. Machner A, Pap G, Awiszus F. Evaluation of quadriceps strength and voluntary activation after unicompartmental arthroplasty for medial osteoarthritis of the knee. J Orthop Res. 2002;20:108-111.

20. Stevens JE, Mizner RL, Snyder-Mackler L. Quadriceps strength and volitional activation before and after total knee arthroplasty for osteoarthritis. J Orthop Res. 2003;21:775-779.

21. Lyons CL, Robb JB, Irrgang JJ, Fitzgerald GK. Differences in quadriceps femoris muscle torque when using a clinical electrical stimulator versus a portable electrical stimulator. Phys Ther. 2005;85:44-51.

22. McDonnell MK, Delitto A, Sinacore DR, Rose SJ. Electrically elicited fatigue test of the quadriceps femoris muscle. Description and reliability. Physical Ther. 1987;67:941-945.

23. Dugan SA, Frontera WR. Muscle fatigue and muscle injury. Phys Med Rehabil Clin N Am. 2000;11:385-403.

24. Fink B, Egl M, Singer J, Fuerst M, Bubenheim M, Neuen-Jacob E. Morphologic changes in the vastus medialis muscle in patients with osteoarthritis of the knee. Arthritis Rheum. 2007;56:3626-3633.

25. Eimre M, Puhke R, Alev K, et al. Altered mitochondrial apparent affinity for ADP and impaired function of mitochondrial creatine kinase in gluteus medius of patients with hip osteoarthritis. Am J Physiol Integr Comp Physiol. 2006;290:R1271-R1275.

26. Tallon MJ, Harris RC, Maffulli N, Tarnopolsky MA. Carnosine, taurine and enzyme activities of human skeletal muscle fibres from elderly subjects with osteoarthritis and young moderately active subjects. Biogerontology. 2007;8:129-137.

27. Glasberg MR, Glasberg JR, Jones RE. Muscle pathology in total knee replacement for severe osteoarthritis: a histochemical and morphometric study. Henry Ford Hosp Med J. 1986;34:37-40.

28. Nakamura T, Suzuki K. Muscular changes in osteoarthritis of the hip and knee. Nihon Seikeigeka Gakkai Zasshi. 1992;66:467-475.

29. Linssen WH, Stegeman DF, Joosten EM, et al. Force and fatigue in human type I muscle fibres. A surface EMG study in patients with congenital myopathy and type I fibre predominance. Brain. 1991;114 (Pt 5): 2123-2132.

30. Berger MJ, McKenzie CA, Chess DG, Goela A, Doherty TJ. Sex differences in quadriceps strength in OA. Int J Sports Med. 2012;33: 926-933.

31. Staron RS, Hagerman FC, Hikida RS, et al. Fiber type composition of the vastus lateralis muscle of young men and women. J Histochem Cytochem. 2000;48:623-629.

32. Wüst RC, Morse CI, de Haan A, Jones DA, Degens H. Sex differences in contractile properties and fatigue resistance of human skeletal muscle. Exp Physiol. 2008;93:843-850.

33. Cairns SP, Knicker AJ, Thompson MW, Sjøgaard G. Evaluation of models used to study neuromuscular fatigue. Exerc Sport Sci Rev. 2005; 33:9-16.

34. Maïsetti O, Guével A, Legros P, Hogrel JY. SEMG power spectrum changes during a sustained $50 \%$ maximum voluntary isometric torque do not depend upon the prior knowledge of the exercise duration. J Electromyogr Kinesiol. 2002;12:103-109. 
Clinical Interventions in Aging

\section{Publish your work in this journal}

Clinical Interventions in Aging is an international, peer-reviewed journal focusing on evidence-based reports on the value or lack thereof of treatments intended to prevent or delay the onset of maladaptive correlates of aging in human beings. This journal is indexed on PubMed Central, MedLine, the American Chemical Society's 'Chemical Abstracts

Service' (CAS), Scopus and the Elsevier Bibliographic databases. The manuscript management system is completely online and includes a very quick and fair peer-review system, which is all easy to use. Visit http://www.dovepress.com/testimonials.php to read real quotes from published authors.

Submit your manuscript here: http://www.dovepress.com/clinical-interventions-in-aging-journal 